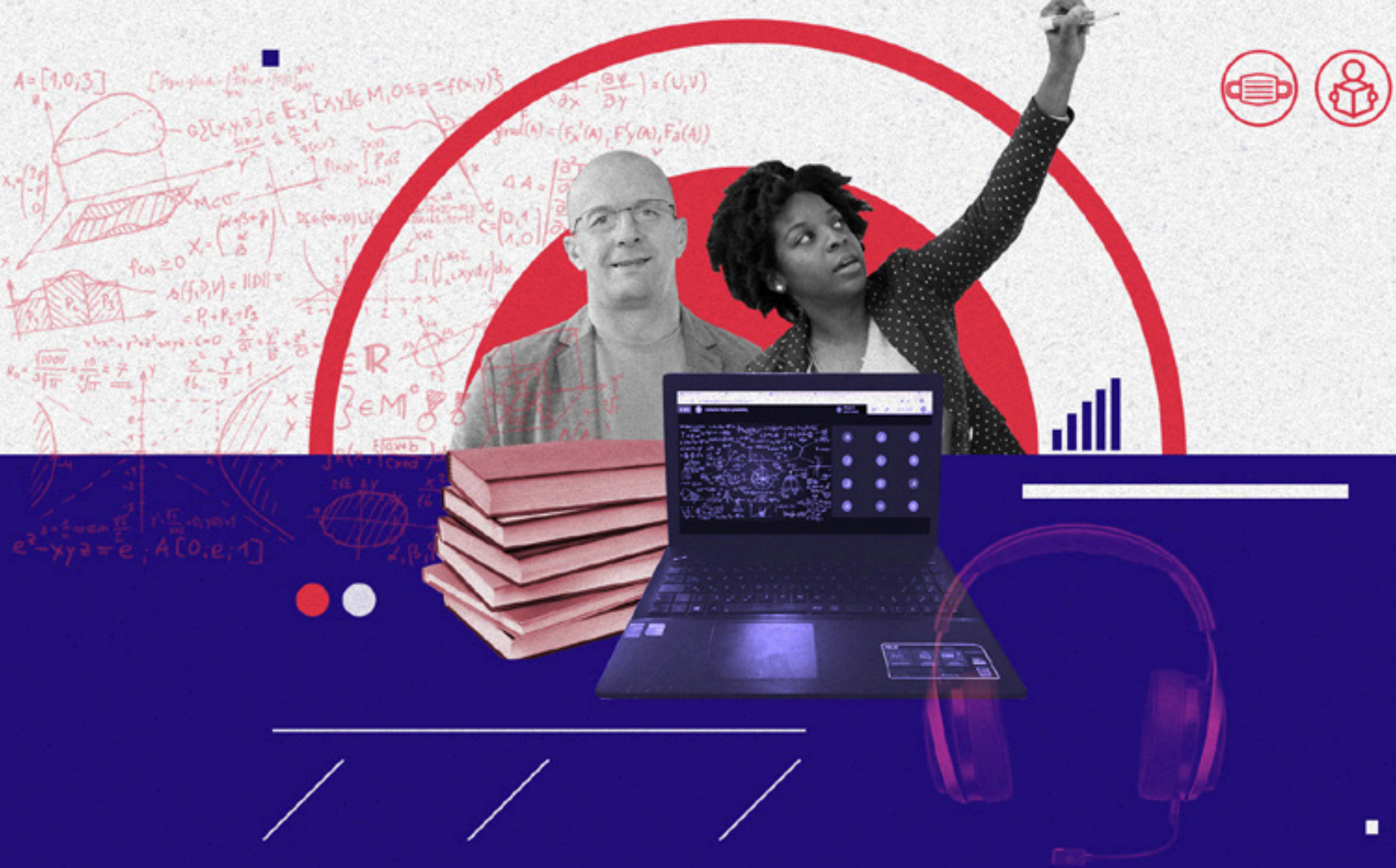

\title{
Valorar la experiencia, volver a la escuela. Aproximaciones a las prácticas de las escuelas oficiales de Bogotá en tiempos de confinamiento
}

VALUE THE EXPERIENCE, GO BACK TO SCHOOL. APPROACHES TO THE PRACTICES OF THE OFFICIAL SCHOOLS OF BOGOTA IN TIMES OF CONFINEMENT

Alejandro Álvarez Gallego; Nylza Offir García Vera ${ }^{3}$; Jhon Henry Orozco Tabares ${ }^{4}$ 


\section{Citar artículo como:}

Álvarez-Gallego, A., Unda-Bernal, M.P., García-Vera, N.O., y Orozco-Tabares, J.H. (2021). Valorar la experiencia, volver a la escuela. Aproximaciones a las prácticas de las escuelas oficiales de Bogotá en tiempos de confinamiento. Educación y Ciudad, n. 41, pp. 19-39. https://doi.org/10.36737/01230425. n41.2021.2633

Fecha de recepción: 9 de abril de 2021

Fecha de aprobación: 4 de julio de 2021

\section{Resumen}

El texto describe algunos de los principales hallazgos del estudio exploratorio titulado: "Valorar la experiencia, volver a la escuela". Para la mayoría de los actores educativos, la educación pos-pandemia reclama examen y previsión, en especial por lo mucho que preocupan sus efectos sobre los vínculos pedagógicos y por el auge ganado por las nuevas tecnologías y sus modificaciones sobre la gramática de la escuela. Trabajos colaborativos, deslocalizaciones, giros y aprendizajes atravesaron la práctica pedagógica de los maestros reafirmando lo mucho que las instituciones educativas pueden cambiar, pero también revalorando el papel social, estratégico y cultural de una escuela presencial imprescindible.

Palabras clave: Escuela; pandemia; práctica pedagógica; maestro; gramática escolar; nuevas tecnologías; presencialidad; derecho a la educación.

\section{Abstract}

The text describes some of the main findings of the exploratory study titled: "Value the experience, go back to school". For most educational actors, post-pandemic education demands examination and foresight, especially because of the great concern about its effects on pedagogical links and the rise gained by new technologies and their modifications on school grammar. Collaborative works, relocations, twist and learning crossed the pedagogical practice of the teachers reaffirming how much educational institutions can change, but also revaluing the social, strategic and cultural role of an essential classroom course school.

Keywords: School; pandemic; pedagogical practice; teacher; school grammar; new technologies; classroom course; right to education.

\section{Resumo}

O texto descreve alguns dos principais achados do estudo exploratório intitulado: "Valorar a experiência, voltar à escola”. Para a maioria dos atores educacionais, a educação pós-pandêmica exige exame e previsão, especialmente pela grande preocupação gerada ante seus efeitos nos vínculos pedagógicos e com o boom das novas tecnologias e suas modificações na gramática da escola. Trabalhos colaborativos, deslocalizações, viradas e aprendizagens atravessaram a prática pedagógica dos professores reafirmando o quanto as instituições de ensino podem mudar, mas também reavaliando o papel social, estratégico e cultural de uma escola presencial imprescindível.

Palavras-chave: Escola; pandemia; prática pedagógica; professor; gramática escolar; novas tecnologias; presença; direito à educação. 


\section{Introducción}

E ste articulo resume los aspectos centrales del estudio exploratorio Valorar la experiencia, volver a la escuela, realizado por la Universidad Pedagógica Nacional y financiado por la Secretaría de Educación Distrital de Bogotá, con el propósito de hacer una aproximación a las prácticas de las escuelas oficiales de la ciudad, durante el período de confinamiento comprendido entre marzo y diciembre de 2020.

Para lograr el propósito, realizamos entrevistas semiestructuradas a profundidad, grupos focales y encuestas a integrantes de las comunidades educativas de zonas rurales y urbanas de todas las localidades de Bogotá. Participaron niñas, niños y jóvenes, maestras y maestros, madres y padres de familia, coordinadores, rectores y orientadores. Buscamos profundizar sobre lo vivido, pero también sobre las lecturas que suscita este tiempo de confinamiento en relación con la escuela presencial anterior a la pandemia. Ahondamos en las lecciones aprendidas, las propuestas de continuidad, de afirmación o de cambio.

Doctor en Filosofía y Ciencias de la Educación. Magister en Estudios Políticos. Licenciado en Ciencias Sociales. Profesor Titular Universidad Pedagógica Nacional, Coordinador Museo Pedagógico Colombiano, Miembro del Grupo de Historia de la Práctica Pedagógica. ORCID: https://orcid. org/0000-0003-2537-4925 Correo electrónico: rizoma. alejandro@gmail.com

Doctora en Cultura y Educación en América Latina . Magíster en Investigación Educativa. Psicóloga. Profesora emérita Universidad Pedagógica Nacional e integrante del equipo coordinador de la Expedición Pedagógica. ORCID: https:// orcid.org/0000-0001-7173-9679 Correo electrónico: pilunda@gmail.com
También consultamos más de cuarenta publicaciones sobre el tema, realizadas en Colombia y otros países de América Latina y revisamos los informes de seguimiento del programa Aprende en Casa de la Secretaría de Educación. Esperamos que este estudio contribuya a animar conversaciones, encuentros y propuestas a partir de la valoración de los caminos recorridos y los aprendizajes que nos dejan.

Destacamos la diversidad como criterio en este trabajo investigativo. Si bien es posible identificar unas tendencias más fuertes que otras, los procesos adelantados en cada colegio y las vivencias de los actores son muy diferentes y dependen de múltiples factores. Los hechos se sucedieron con mucha rapidez, no fue igual lo que se hizo al inicio de la pandemia bajo el supuesto de que pronto se volvería a la presencialidad, lo que sucedió a medida que se constataba que el confinamiento persistía, o cuando se aceptó que pasaría un tiempo considerable antes de volver a la escuela. Por esto, al marcar ciertas tendencias en la presentación de los hallazgos, no intentamos hacer generalizaciones. Por el contrario,

Doctora en Educación por el Doctorado Interinstitucional en Educación de la Universidad Pedagógica Nacional. Magister en Educación. Licenciada en Pedagogía. Especialista en la Enseñanza del Español y la Literatura. Profesora de la Facultad de Educación de la Universidad Pedagógica Nacional. ORCID: https://orcid.org/00000002-7232-4210 Correo electrónico: nylzao@yahoo.es

Magíster en Educación. Licenciado en Filosofía y Letras. Profesor de la Facultad de Educación de la Universidad Pedagógica Nacional. Integrante del Grupo de Historia de la Práctica Pedagógica. ORCID: https://orcid.org/0000-00023807-1803 Correo electrónico: aulamundo@yahoo.com 
advertimos sobre la necesidad de reconocer multiplicidades, singularidades y complejidades.

De entrada, es necesario delimitar los alcances de este estudio. Aun cuando habíamos previsto estrategias específicas para aproximarnos a aquellos sectores poblacionales que no han tenido acceso a las herramientas de comunicación telemáticas, las circunstancias de la pandemia lo impidieron. Buscamos saber de ellos a través de las voces de maestros, orientadores y directivos, incluso de otras familias, pero el primer llamado de este estudio es sobre la imperiosa necesidad de mejorar la conectividad, un asunto que se ha querido atender desde las escuelas con grandes esfuerzos que a todas luces han sido insuficientes. Se requiere definir políticas y comprometer acciones de Estado que, al menos, contribuyan a mitigar los efectos de este aspecto que algunos de los autores consultados llamaron "nuevas desigualdades".

\section{La escuela se movilizó, se vio interpelada por la agudización de la pobreza y buscó restablecer los vínculos}

En un tiempo de incertidumbre para el que nadie estaba preparado, la comunidad educativa enfrentó los desafíos, a veces de manera conjunta y otras por separado, pero no se inmovilizó. Por el contrario, exploró múltiples alternativas para enfrentar la situación.

El primer momento estuvo caracterizado por la acción de directivos y maestros, quienes asistieron al colegio, se organizaron y elaboraron "guías" para realizar desde casa, con la expectativa de que volverían a encontrarse después de quince días de confinamiento preventivo obligatorio. A medida que pasó el tiempo, cuando se entendió que la situación se prolongaría, se dieron a la tarea de contactar y comunicarse con cada uno de los niños y sus familias para saber cómo estaban siendo afectados por la emergencia sanitaria y trazar rutas de acción. No se tenían teléfonos ni datos de contacto, existía una norma más o menos generalizada, tácita en algunos casos, según la cual los maestros debían evitar este tipo de comunicaciones para prevenir situaciones de acoso. Tampoco tenían acceso a las bases de datos dado que las instalaciones de los colegios se encontraban cerradas y, según nuestras encuestas, casi el 50\% de las familias tenían dificultades de comunicación. Poco a poco, a partir de unas primeras conexiones con aportes de madres, padres y de los mismos niños, se fueron ubicando los estudiantes y realizaron los primeros "diagnósticos", tal como los llamaron algunos. Desde entonces, la escuela se vio movilizada por una profunda sensibilidad ante las evidencias de pobreza extrema, el hambre, el desempleo, la enfermedad, el impacto emocional por la pandemia y el aislamiento. Si bien las encuestas a familias y niños no dieron cuenta de estas situaciones, aspecto que registramos con extrañeza, éstas si fueron muy notorias en las entrevistas y los grupos focales.

Los profesores adelantaron campañas de solidaridad, hicieron colectas con sus propios recursos para mitigar el hambre o garantizar condiciones mínimas de comunicación como las tarjetas SIM o los celulares, para poder vincularse con los más aislados. Pronto se dieron cuenta de sus limitaciones, pocas familias habían recibido apoyo de los gobiernos nacional o distrital, la magnitud de esta problemática quedaba fuera de su alcance. Al final se tomó una 
decisión, centrarían todos sus esfuerzos en lo que la escuela sabe hacer: realizar su trabajo pedagógico, fortalecer los vínculos, enseñar. Al decir de Gabriela Diker, a partir de la evaluación de la continuidad docente en Argentina: "Si bien sabemos que la escuela no alcanza a resolver el problema de la desigualdad social y que incluso contribuye a reforzar, está claro que sin escuela la desigualdad se profundiza." (Diker, 2020)

En adelante, los maestros se vieron atravesados por el deseo de incluir a todos los estudiantes, interpelados por unas realidades que ahora se hacían más visibles y por sentimientos de impotencia ante las ausencias y las limitaciones propias de enseñar desde la distancia. Algunos niños se habían devuelto a sus lugares de origen, otros se habían ocupado en actividades familiares que comprometieron sus tiempos de estudio, no contaban con dispositivos ni conexión a internet o, si los tenían, debían compartirlos con otros integrantes de la familia.

Encontramos maestros que dedicaron considerables esfuerzos, a medida que se iban introduciendo y aproximando a distintas tecnologías, a diseñar simultáneamente múltiples estrategias en función de las circunstancias diversas de conexión, acceso a aparatos, tiempos y particularidades de los estudiantes. Elaboraron guías físicas que entregaban en el colegio o enviaban por correo urbano, guías digitales mediante correo electrónico, grabaciones a través de los celulares o plataformas, clases asincrónicas por plataformas escolares para quienes no alcanzaban a coincidir en los mismos tiempos y clases sincrónicas para aquellos que disponían de tiempo, conexión y aparatos.
Así pues, los maestros no solo se vieron desafiados por las viejas desigualdades, agravadas ahora con la pandemia, también adelantaron iniciativas con el propósito de reducir los efectos de las "brechas digitales de primer orden" (Narodowski y Campetella, 2020), esto es, las oportunidades desiguales de conectividad y acceso a dispositivos tecnológicos. Evidentemente se trata de una responsabilidad que no le cabe solamente a la escuela, por eso requiere políticas públicas que garanticen el derecho a la educación para todos.

Numerosos integrantes de las comunidades educativas contribuyeron con sus acciones a garantizar la continuidad de la escuela. Aún así, han sido los maestros el "andamio principal de la educación", como lo han reconocido en Chile los resultados de la encuesta \#Vinculando aprendizajes (Fundación Chile, 2020) y Rodríguez, Estrada y Naranjo (2020) en su análisis de lo sucedido en Medellín. Los maestros estuvieron centrados en la tarea de restablecer la comunicación, fortalecer los vínculos con los estudiantes y sus familias. Además, se ocuparon de responsabilidades que, aún cuando no les correspondían, resultaban indispensables para adelantar su tarea de enseñar, enfrentándose a sus propios límites, preparando materiales, ideando y flexibilizando sus formas de trabajo.

En esto coinciden maestros, estudiantes, orientadores, directivos y padres de familia encuestados. Por ejemplo, un alto porcentaje de madres y padres $(84,34 \%)$ afirmó que los maestros trabajaron con sus hijos semanalmente, la mayoría de ellos entre 4 o 5 días. Sin embargo, también reportaron que se presentaron casos de estudiantes que fueron aten- 
didos esporádicamente $(0,68 \%)$, o nunca $(6,12 \%)$, datos que resultan preocupantes pues a estos niños no se les garantizó el derecho a la educación.

Entre los aportes de los maestros, los estudiantes identificaron en su orden: ayudarles a ser mejores personas, compañeros, hijos, amigos, estudiantes (76\%); apoyo y acompañamiento durante las situaciones vividas (69\%); adquisición de nuevos conocimientos, saberes y habilidades (59\%) y desarrollar la creatividad y la imaginación (57\%). Lo relevante aquí es que, desde la perspectiva estudiantil, el maestro jugó un papel fundamental en el tema socioemocional y psicológico en las crisis que vivieron ellos y sus familias, pero no descuidó su tarea formativa en su dimensión cognitiva. Los padres de familia coincidieron en términos generales con estos resultados, pero registraron en primer lugar los aportes a la creatividad e imaginación de sus hijos y muy pocos incluyeron el acompañamiento frente a las situaciones vividas.

Según numerosos autores se ha producido una reconfiguración del derecho a la educación. No solo se agudizaron las desigualdades previas, que ya se reflejaban sobre los sistemas educativos y en el contexto social, sino que se superpusieron nuevas desigualdades que fueron visibilizadas por la pandemia (Pinau y Ayuso, 2020; Cardini, Bergamaschi, D’Alessandre, Torre y Ollivier, 2020). Aparece entonces un reclamo por el papel protagónico que se espera del Estado en medio de esta crisis. El evidente atraso tecnológico de nuestras instituciones educativas y la ausencia de oportunidades de conexión de las familias más vulnerables no admite dilaciones, requieren una pronta y efectiva intervención.
Autores como Narodowski y Campetella (2020) identifican diversos tipos de brechas digitales: las de primer orden referidas al simple acceso a las tecnologías de la información y la comunicación, las de segundo orden enfocadas al uso más selectivo y formativo de los contenidos, y las de tercer orden dirigidas a identificar las escuelas que le dan a las tecnologías un enfoque innovador. Esta taxonomía también puede resultar insuficiente, vale la pena retomar el planteamiento del Observatorio Interuniversitario de Sociedad, Tecnología y Educación OISTE - de Argentina, acerca de la importancia del Estado "como garante de la reducción de las brechas sociales, digitales y educativas".

\section{Superposición de tiempos y espacios laborales, educativos y domésticos}

Cuando se suspende la escuela presencial, lo primero que se reconfigura es la espacialidad y los tiempos de los hogares, especialmente de los niños, los maestros y sus familias. La escuela entra a la casa y con ello se modifican y trastocan rutinas, tiempos, tareas y funciones. En ocasiones esto significó la oportunidad de compartir, conocerse, disfrutar más la familia, en otras se percibió el afán de supervivencia, la vulnerabilidad y la soledad.

Se produjo una superposición de tiempos y espacios laborales, educativos y domésticos, con implicaciones para todos, pero especialmente para las mujeres. Con lo anterior se intensifica la cultura patriarcal que aún persiste, las madres asumieron en buena parte la responsabilidad del cuidado y las maestras se vieron 
en la necesidad de atender simultáneamente, en un mismo momento y lugar, la atención y el estudio de los hijos, el cuidado de los más pequeños, las labores domésticas, las prácticas pedagógicas con sus estudiantes:

Hay momentos que uno está conectado y el niño lleva una hora sin hacer nada, sin bañar, no he hecho el almuerzo, tengo que revisar los trabajos que me envían. Hay momentos en que el trabajo es tanto y se acumulan tantas cosas que a veces se desborda todo lo que uno había organizado en temas de rutinas (Entrevista maestra Nathalie Perez, citada por Álvarez, Unda, García y Orozco, 2021, p. 22).

Desde una perspectiva pedagógica, quedarse en casa, no diferenciar el espacio familiar del escolar, restringe una de las tareas centrales de la escuela: posibilitar que las nuevas generaciones accedan a otros mundos, reconozcan y a la vez tomen distancia de los referentes familiares o locales para codificar de manera más compleja la realidad.

Podemos afirmar que en este tiempo se alteró y reconfiguró la gramática propia de la escuela: cambiaron los tiempos, la disposición de los cuerpos, los modos de relacionamiento, los contenidos, los instrumentos, las formas de trabajar. Las prácticas fueron otras, múltiples y muy variadas. Se dieron casos de organización de los grados por ciclos y niveles, en ocasiones se pasó de cuatro a tres períodos en el año, o de tres a dos. Las jornadas y las modalidades de trabajo de los maestros cambiaron, debían estar conectados de muchas maneras con sus estudiantes y en horarios muy flexibles y extensos.

\section{Prevalencia de las guías y develamiento de algunas problemáticas de la escuela presencial}

En el estudio se destaca la prevalencia de las guías elaboradas por los maestros de cada institución, por cuatro razones principales: (i) fue la primera estrategia de trabajo propuesta por los colegios y por la misma Secretaría de Educación, para atender una situación que parecía transitoria; (ii) en contraste con otras tecnologías utilizadas, las guías se revelaron como una herramienta privilegiada para sostener los vínculos y realizar el trabajo con los estudiantes que no tenían acceso a través de otros dispositivos; (iii) el uso de las guías posibilitó que la misma comunidad educativa pusiera al descubierto aspectos problemáticos de las prácticas escolares anteriores a la pandemia, se generaron debates, se hicieron replanteamientos y se adelantaron iniciativas de modificación y cambio; (iv) al final, fueron altamente valoradas por estudiantes y padres de familia, al punto de identificarlas como una de las prácticas con la que desearían seguir contando en la escuela post-pandemia.

Hacemos notar que el término "guía" no es unívoco, hace referencia a una multiplicidad de prácticas diferenciadas en sus fines, su estructura, su disposición y sus contenidos, incluso en presentaciones y diseños. Existen muchas maneras de entender lo que es una guía y en ellas se puede encontrar las 
más variadas propuestas ${ }^{5}$. Sin embargo, desde el primer momento se hizo sentir con mucha fuerza el agobio y la impotencia de niños y padres de familias que se resistían a realizar un trabajo que los abrumaba, no lo entendían, o no contaban con el tiempo requerido. La expresión ¡Nos tostamos! de un estudiante que participó en los grupos focales, expresa acertadamente ese sentimiento.

Según el informe del período marzo-abril de la estrategia Aprende en Casa (SED, 2020), ante la imposibilidad de los estudiantes y sus familias de seguir los ritmos de trabajo y las extensas jornadas de trabajo requeridas por los maestros para la elaboración y revisión de guías, se vio la necesidad de hacer ajustes a las acciones propuestas. Las dificultades de los estudiantes para resolver las guías y talleres formulados obedecían a varios factores: (i) habían sido diseñados como si estuvieran en clases presenciales y requerían acompañamiento de los maestros; (ii) no se había fortalecido previamente el trabajo autónomo de los niños en los colegios; (iii) no permitían atender diferencialmente a los niños de cada grupo; (iv) existían "cuestiones estructurales" que limitaban las acciones pedagógicas que los maestros buscaban orientar a través de estos materiales, por lo cual se hacía necesario incluir familias y cuidadores.

Con la prolongación de la cuarentena, las guías se fueron transformando. Frente al clamor de las familias y los estudiantes por la dificultad para entender-

\footnotetext{
En el micrositio de la estrategia Aprende en Casa de la SED se encuentra un número considerable de guías, elaboradas por maestros de distintos colegios de la ciudad, que se convierten en un soporte valioso para realizar un estudio sobre su diversidad. https://www.redacademica.edu.co/ estrategias/aprende-en-casa
}

las y la cantidad de trabajo que se exigía en ellas, se propuso reducir las tareas, priorizar objetivos, agrupar y articular contenidos, incluso los maestros se enfocaron en el diseño, la presentación y la estética de sus materiales. Algunos maestros señalaron su preocupación por la autonomía que tendrían para producir los materiales, pues hubo casos en los que el colegio envió materiales estandarizados. En otros casos, se mantuvieron las posturas más conservadoras, el desconocimiento y tratamiento vertical frente a los estudiantes, la aplicación de fórmulas ya conocidas, la imposibilidad de movilizar propuestas novedosas.

Al final, junto con las guías físicas se experimentaron de manera simultánea o sucesiva, diversas actividades en el ejercicio pedagógico. Las más recurrentes fueron: sesiones grupales a través de medios virtuales (88\%), envío de tareas y guías a través de WhatsApp (86\%), envío de tareas y guías a través de correo electrónico $(85,5 \%)$, envío de tareas y guías en medios físicos (75,5\%), envío de tareas y guías mediante audios o videos $(71,5 \%)$, sesiones individuales por medios virtuales (62\%), proyectos virtuales y seguimiento de los mismos (56\%), envío de tareas y guías a través de llamadas telefónicas (47,5\%). El mayor porcentaje de maestros (65\%) mencionó las llamadas telefónicas como el recurso más utilizado para contactar a los estudiantes que no tenían conectividad. 
Los replanteamientos y cambios no se hicieron esperar. A veces junto con otros maestros de la misma institución, otras con el apoyo decidido de coordinadores o rectores y también de manera solitaria, un buen número de maestros (no todos, por supuesto) se dieron a la tarea de interrogar las propias prácticas, redefinirlas, atreverse a ensayar. Otros aspectos de la escuela presencial fueron problematizados por la comunidad educativa durante este período, así por ejemplo: la compartimentación de la enseñanza, la saturación de contenidos y de tareas, las dificultades para atender las particularidades, las circunstancias y procesos desiguales de los estudiantes en un mismo grupo.

Surgieron controversias, se hicieron evidentes las inquietudes de maestros ante el dilema ético que enfrentaron debido a que al dedicarse a aquellos estudiantes que podían conectarse, estarían contribuyendo a profundizar las desigualdades, pues otros quedaban excluidos.

\section{Educar sin cuerpos, el predominio de las tecnologías}

Tal vez el tema de las tecnologías ha sido uno de los más comentados desde que se inició la pandemia, pero poco se ha dicho sobre sus implicaciones pedagógicas. Este estudio constata el tránsito de una utilización tímida y marginal de las tecnologías en las escuelas, a una relación que se intensificó, se multiplicó y diversificó, para garantizar la continuidad del sistema educativo. La tecnología permitió entre otras: restablecer y sostener los vínculos, adelantar la tarea educativa y realizar la gestión académico - administrativa de las instituciones.
Sorprendió la capacidad de la comunidad educativa para enfrentar el atraso tecnológico en el que se encontraba la escuela, incluidas las familias. Incluso se superaron las dificultades de los niños que habían sido considerados "nativos digitales", pues algunos de ellos evidenciaron la distancia entre interactuar en redes sociales y saber utilizar las herramientas virtuales para explorar, investigar, interactuar académicamente, aprender y construir conocimiento.

Ante la necesidad de alternativas que permitieran mayor acceso de los estudiantes y hacer más atractiva la enseñanza, los maestros hicieron grandes esfuerzos, vencieron prejuicios sobre sus propias capacidades, temores y miedos, exploraron y se atrevieron a ensayar. A veces estuvieron acompañados por los colegas que tenían mayor dominio de las herramientas virtuales, como los profesores de tecnología y matemáticas, o de aquellos que habían realizado estudios de posgrado en las universidades.

La gama de herramientas tecnológicas utilizadas fue muy amplia: WhatsApp, plataformas de encuentro, correos electrónicos, páginas web, redes sociales, podcasts, programas de diseño, entre otras. La plataforma Teams que la Secretaría de Educación puso a disposición de los colegios a partir de junio de 2020, posibilitó la realización de encuentros sincrónicos. Aquí se desataron sentimientos agridulces, las emociones por volverse a ver y el dolor por las ausencias de niños que no tenían posibilidades de conexión o los tiempos requeridos para participar a las horas previstas. Formas de exclusión que, como ya hemos dicho, profundizan las desigualdades ya existentes.

Muchos maestros aprendieron a hacer videos, fotografías, podcasts, a planear jornadas sincrónicas con 
trabajos asincrónicos, así pues, sus prácticas se vieron enriquecidas. Otros tomaron distancia, interrogaron sus efectos y decidieron no usarlas:

Cada vez se habla más de soluciones tipo plataformas pedagógicas, diseños de contenido, entornos virtuales de aprendizajes, trabajo digital colaborativo, la digitalización se nos vende como la solución a los problemas de equidad social, pero obviamos sus grandes problemas: eliminan la espesura del lenguaje y sobre todo centraliza lo que se produce en dispositivos de control que son la forma más efectiva de gobernarnos a distancia (VV.AA., 2020, p. 40).

Para varios de los directores educativos el tema de la formación para el manejo de las tecnologías se tornó estratégico, hablaban de: "fortalecimiento en el manejo de dispositivos", "manejo de herramientas tecnológicas, capacitación en uso de herramientas tics", "alfabetización digital". Uno de ellos afirma directamente "dejar de plantear dificultades de tecnología y sus aplicaciones en términos de problema y trabajar para su solución".

Las nuevas conexiones entre escuelas y familias produjeron efectos sobre las prácticas. Los maestros "entraron" a las casas a través de las pantallas y los padres de familia accedieron directamente a las actividades que estos realizaban con sus hijos. No pocos maestros se impactaron con las precariedades y vulnerabilidades extremas de sus estudiantes, se sorprendieron y se dolieron con los contrastes económicos entre las familias de los niños de un mismo grupo.

Mientras algunos padres cuestionaron a los maestros por sus prácticas, otros comprendieron la importancia de actividades que antes no valoraban (así por ejemplo los juegos, en lugar de exigir planas o tareas convencionales, en el caso de preescolar y los primeros grados). En general, se produjo una revalorización de la escuela y del maestro. El cuidado, la dedicación, el seguimiento y la atención que les dedicaron a ellos y a sus hijos durante este tiempo, ha sido una de las características de estas prácticas que los padres proponen continuar en la escuela pos pandemia. También destacamos los testimonios de madres y padres de familia que por haber tenido la oportunidad de acompañar las actividades y los aprendizajes de sus hijos, contemplaron la posibilidad de continuar sus estudios propios.

La introducción en las tecnologías posibilitó a maestros de todas las edades, a niños y padres, descubrir posibilidades que antes no conocían, acceder a herramientas que entusiasmaron y motivaron la imaginación: "El manejo de redes y computadores, eso toca hacerlo, que tengamos acceso y conocimiento del uso de distintas plataformas y programas que nos ayuden a manejar y comunicar la información de una manera más acorde. No hay que hacerle el feo a la tecnología" (Entrevista Romero-Contreras, 2021).

En términos generales, las tecnologías fueron abordadas como herramientas que facilitan el acceso a la información, que enriquecen, complementan o amplían los alcances de las propuestas pedagógicas de los maestros, pero no las subordinan ni sustituyen. También se presentaron posturas críticas con alto nivel de argumentación, sobre las afectaciones que pueden tener ciertas plataformas en las prácticas educativas:

Entonces empecé a ver que las multinacionales se meten en el fondo del currículo, en la estructura [...], cómo los medios juegan con las luces, los logos y los videos y cómo esto se vuelve un lenguaje generali- 
zado, necesario, la forma en que obligan a las clases populares a que aprendan un lenguaje que incluyó a la escuela (Entrevista maestro Camilo Romero, citado por Álvarez, Unda, García y Orozco, 2021, p. 71).

Desde esta perspectiva, las tecnologías alteran, limitan, ponen condiciones en función de intereses que actúan y atraviesan las prácticas, es decir, se presentan como un nuevo actor en las prácticas educativas. En ningún caso registramos apuestas en favor de la sustitución del maestro por las tecnologías, una perspectiva que, desde antes de la pandemia, ha sido agenciada por algunos organismos transnacionales.

Fueron muy diferentes las experiencias de los estudiantes en relación con las tecnologías y a medida que fueron ocurriendo, se constituían en un desafío para los maestros. Mientras algunos conseguían sorprenderse y entusiasmarse, en un alto número de casos, según los resultados de las encuestas, no lograban concentrarse, no entendían, se dispersaban, se distraían.

Las percepciones de los padres de familia a este respecto fueron variadas. Para algunas familias con el uso de estas tecnologías los maestros renovaron sus metodologías y lograron un mayor interés y motivación. Insistieron en que los hijos ganaron en autonomía, hubo preocupación por el rendimiento escolar de sus hijos, o por verlos permanentemente conectados. En lo que sí coincidieron fue en la realización de reuniones de padres de familia a través de plataformas virtuales como un gran acierto, es una opción que quieren conservar al volver a la presencialidad, pues les permite asistir desde sus casas $\mathrm{y}$ han tenido mejores condiciones para participar y tener más información.

\section{Prácticas pedagógicas: variaciones, giros, deslocalizaciones}

Lo que ocurrió después de la fase inicial, caracterizada por una amplia movilización de la comunidad educativa para restablecer contactos, liderar acciones de solidaridad con las familias más vulnerables y recomponer los vínculos, fue que la atención se centró en idear, ensayar, poner a prueba, experimentar modos de trabajo que permitieran adelantar procesos de enseñanza y formación en las nuevas circunstancias, desde la distancia, con herramientas que muy pocos conocían y la desestructuración de aspectos centrales de la gramática escolar (Álvarez-Gallego, 2020).

Los sentimientos de compromiso, esperanza y decisión de sacar adelante la escuela que animaron todos los esfuerzos de solidaridad y restablecimiento del vínculo educativo, se vieron opacados por la evidencia de que la escuela, por sí sola, no consigue asegurar el derecho a la educación de todos los estudiantes. Hoy sabemos que durante el 2020 en Bogotá, el 2,5\% de los estudiantes públicos y privados desertó y el $52 \%$ de los colegios oficiales reportó 
dificultades de acceso a sus actividades desde casa ${ }^{6}$. Aun así, resaltamos la capacidad de superponerse, el entusiasmo, la decisión, la esperanza, la necesidad de darle continuidad a la escuela.

Algunos maestros encontraron en las tecnologías herramientas favorables para niños en condición de discapacidad, en particular porque permitían "explorar algunas de sus habilidades, por ejemplo, el niño especial que no sabía leer y escribir, pero ahora prende su computador, se conecta y participa en clase" (Entrevista López, 2021). También ocurrió con niños que en la presencialidad se veían opacados, no se atrevían a participar o eran objeto de acoso escolar y ahora, contrario a lo que esperaban algunos maestros, se destacaron por su interés y participación.

Se encontró una amplia gama de experiencias que nos hablan de la riqueza pedagógica y también de la autonomía que tienen las escuelas. No se partía de cero, los maestros traían unos consolidados, trayectorias propias del trabajo en redes que se convirtieron en un referente para enfrentar con imaginación las nuevas circunstancias, teniendo en cuenta sus propios enfoques y perspectivas.

Es claro que los maestros respondieron con creatividad al desafío de la educación no presencial, lo cual ya resulta muy interesante. También queremos des- tacar que al adaptarse al nuevo contexto enriquecieron sus propias prácticas. El 59\% de los maestros que respondieron la encuesta consideraron que mejoraron en el diseño y la selección de contenidos y materiales de apoyo; 53\% optimizaron la preparación y planeación de las clases; $50 \%$ aumentaron el trabajo colaborativo con los colegas del área, del nivel, o del colegio; y 47,5\% incrementaron el uso de nuevas metodologías y estrategias pedagógico-didácticas innovadoras.

Vale la pena destacar que el tema emocional ocupó un lugar preponderante inicialmente, con propuestas que buscaban contribuir al bienestar de los niños y de sus familias ante la nueva situación, de tal manera que pudieran expresar y comprender mejor lo que estaba sucediendo, según los niveles educativos. Al poco tiempo de trabajar estos asuntos desde cada área de conocimiento, se evidenció la saturación que estaban produciendo, lo que ameritaba la diversificación y selección de otros contenidos.

Los orientadores escolares, en su mayoría mujeres, tuvieron que sobreponerse a sus propios quiebres emocionales (ansiedades, temores diversos e incluso depresiones difíciles de manejar) y terminaron ampliando los alcances de sus acciones y servicios para atender a los maestros y a las familias, viendo revalorado su papel en la escuela.

Según la encuesta realizada por ProBogotá, la Universidad de Los Andes y la Escuela de Gobierno Alberto Lleras Camargo, aun cuando la mayoría de los estudiantes permaneció matriculada en el 2020, 34.934 salieron del sistema y dejaron de estudiar durante la pandemia, de los cuales el $68,4 \%$ son niñas. Por otra parte, el $52 \%$ de los estudiantes de colegios oficiales y el $49 \%$ de estudiantes de estratos 1 y 2 reportó haber tenido dificultades de acceso a las actividades escolares. 
A continuación, presentamos los principales giros o "deslocalizaciones" que podemos destacar a partir de los relatos sobre las prácticas pedagógicas.

a. Flexibilización y articulación de contenidos y formas de enseñar: de la dispersión a la definición de prioridades.

Estaba visto que, al darle continuidad a las prácticas anteriores a la pandemia, los estudiantes, sus familias y los mismos maestros, se verían desbordados por la cantidad de tiempo y los esfuerzos requeridos para la preparación de materiales y la revisión de los trabajos. Según las materias a cargo debían preparar actividades para un número considerable de grupos. El esfuerzo era mayor para quienes tenían menos intensidad horaria por materia (hasta 22 grupos con una intensidad de una hora semanal).

Si bien algunos intentaron continuar trabajando como lo hacían antes, otros decidieron priorizar objetivos con el fin de evitar la saturación de actividades y tareas puesto que ahora sabían cuánto se habían visto afectados por el cúmulo de trabajo, tanto los niños como sus padres, amigos o vecinos que habían intentado ayudarles.

A partir de lo anterior, seleccionaron contenidos, definieron y redujeron el número de actividades y prepararon los materiales requeridos. Esto supuso altos grados de flexibilización para adecuar a las nuevas circunstancias procesos pedagógicos propios de la escuela presencial que suponen la cercanía del maestro, el acompañamiento, la valoración de los avances, la corrección de los errores cuerpo a cuerpo, casi de manera inmediata. Este es uno de los aspectos que más se añoran y valoran de las prácticas pedagógicas presenciales y no pueden ser sustituidos por las mediaciones tecnológicas.

El tipo de prácticas realizadas comúnmente, se caracterizó por hacer parte de un proceso (41\%), en lugar de actividades aisladas, puntuales, o desarticuladas, algo que pedagógicamente ha sido siempre deseado. Se destacaron los talleres de lectura de textos, libros, revistas y otras fuentes de consulta (71\%), escritura de textos académicos (ensayos, resúmenes, comentarios, reseñas) (68\%), productos audiovisuales (audios, videos, secuencias de imágenes) (63\%), exposiciones en video o audio a través de encuentros virtuales (59\%), productos audiovisuales resultado de un proceso (56\%), cuestionarios sobre un tema específico (53\%), escritura de cuentos, poemas, crónicas, diario, textos para periódico (41\%), lo cuál habla de la versatilidad y la recursividad de los maestros.

b. Del trabajo individual al trabajo colaborativo en la escuela

El trabajo por equipos implicó largas jornadas de planeación y organización, no exentas de discusiones importantes y a veces agotadoras en los consejos académicos, consejos directivos, reuniones de área, comités de evaluación, entre otras instancias.

Pero la priorización de objetivos, de contenidos y de actividades de manera individual, por parte de cada maestro, no resultaba suficiente para atender la magnitud de la problemática presentada. Era necesario conformar grupos, acordar cuáles de sus propósitos formativos compartían y consideraban prioritarios, romper con la separación entre áreas y materias, definir formas de trabajo conjunto, inclu- 
sive criterios y formas de evaluar. Surgieron otras formas de organización del trabajo escolar.

En algunos colegios se pasó del trabajo individual al trabajo colectivo de manera paulatina, a medida que se reunían virtualmente ya sea para sentirse acompañados, para conocer cómo estaban enfrentando la situación otros colegas, para resolver conjuntamente las dificultades de las prácticas. En otros casos ya se tenían experiencias de trabajo conjunto, pero se intensificó durante este período: "la virtualidad se ha prestado para integrar mucho más los equipos de trabajo que teníamos en la presencialidad. Entonces se ha notado más el tema de que los profes estemos construyendo entre todos estrategias, poniéndonos de acuerdo" (Entrevista López, 2021).

Parece ser que estas prácticas han sido frecuentes en varios colegios, especialmente entre maestras de preescolar, según los resultados de las entrevistas. Se encontraron casos de maestros que intentaron dinámicas de articulación entre distintas materias o áreas, aportando sus experiencias previas de trabajo en redes pedagógicas y aun cuando no siempre lo lograron, las dificultades encontradas se convierten en una oportunidad para profundizar las lecturas críticas sobre la cultura escolar.

Lo cierto es que durante este tiempo surgieron, se exploraron o se intensificaron formas de trabajo conjunto en la escuela. Para algunos maestros la situación de confinamiento abrió esta posibilidad, que en otras circunstancias no se habría dado. Ha sido una de las experiencias más valoradas por parte de los maestros, pues contribuyó a romper con la insularidad y tuvo importantes efectos sobre sus prácticas pedagógicas. c. Interrogantes sobre la organización del currículo por materias

Se destacaron las experiencias de flexibilización curricular y de implementación de proyectos pedagógicos que interrogaban la organización disciplinar por materias:

Entendimos que el currículo, formulado en las mallas curriculares, no tenía vigencia, eso se desbarató y fue una discusión con compañeros que pensaban que en pandemia podían seguir haciendo clase común y corriente y llenar esa lista de contenidos como si nada estuviera pasando (Entrevista R. A. Hernández, citada por Álvarez, Unda, García y Orozco, 2021, p. 57).

Surgieron con frecuencia interrogantes sobre la falta de consistencia entre las "mallas curriculares" definidas institucionalmente y las prácticas pedagógicas que se llevaban a cabo. Se cuestionaba la organización disciplinar y la estructura jerárquica entre asignaturas:

La escuela presencial aún tiene muchas estructuras disciplinares y prácticas del siglo XIX [...] Algo que ya está mandado a recoger, pero que sigue muy vigente, el tema de las asignaturas. Cada maestro se ubica en una isla y entonces el profe de matemáticas piensa que su clase es mejor que la tuya porque tú eres de ética y religión. Con la cantidad de guías que se generaron de cada asignatura, los chicos estaban destruidos" (Entrevista Guzmán, 2021).

Maestros de diferentes especialidades acordaron trabajar por "campos de pensamiento" o inspirados en determinadas problemáticas sociales y culturales cuyo abordaje se vería enriquecido por la articulación de saberes. 
Existen hoy diversidad de experiencias de proyectos "transversales" que articulan áreas de saber y propuestas de pedagogías por proyectos. Algunos de estos conocimientos ya traían una trayectoria en la misma escuela y tuvieron la capacidad de ajustarse a las nuevas circunstancias con altas dosis de imaginación:

[...] nos parecía durísimo el tener que renunciar a encontrarnos, a volvernos a referenciar en un espacio, en un sitio para poder desarrollar una propuesta de carnaval como se había estado haciendo [...] Se renuncia un poco a lo lúdico, a la interacción social real por la virtualidad, que son otros espacios, otros metalenguajes totalmente diferentes y estábamos explorando, esto es lo más interesante, a ninguno se nos había ocurrido un carnaval virtual, pues este se caracteriza por el encuentro con el otro (Entrevista Rosero, 2021)

\section{d. Flexibilización de las prácticas evaluativas}

Las experiencias respecto a las formas de evaluar que surgieron para atender las circunstancias particulares de la pandemia, han generado un conjunto de debates y replanteamientos sobre qué evaluar, cómo hacerlo y con qué criterios.

Evidenciamos una tendencia a flexibilizar las prácticas evaluativas desde los aspectos a considerar, las exigencias en los trabajos y los exámenes, hasta la forma de calificar y los intentos de pasar de lo cuantitativo a lo cualitativo. Ha sido uno de los temas sensibles que genera grandes inquietudes, preocupación por las implicaciones pedagógicas y sus efectos sobre la formación y la enseñanza. Es uno de los temas que ha sido objeto de interesantes debates pedagógicos, un punto de inflexión importante si se piensa en los intentos que durante años se han hecho para transformar la evaluación.
Se destacan la flexibilidad, el énfasis en el acompañamiento y el seguimiento a los estudiantes, tener en cuenta las situaciones que se les presentan, incluso el compromiso de sus familias, así como momentos y ajustes en las escalas de medición. Podemos decir que se produjo una flexibilización de las prácticas evaluativas, lo cual parece consistente con los desplazamientos mencionados anteriormente en relación con las prácticas: "hemos aprendido a flexibilizar las mallas, a mirar otras formas de evaluación de los estudiantes, a valorar otro tipo de esfuerzos, de características, de condiciones, que son aspectos necesarios en evaluación" (Entrevista López, 2021).

Algunos maestros reportaron que los estudiantes que no tuvieron conectividad "simplemente perdieron", debido a que el colegio buscó comunicarse con ellos pero "ante la falta de señales, tuvieron que dejarlos". También se dieron casos de flexibilización de la evaluación debido a consideraciones relacionadas con las implicaciones sociales y emocionales de los niños, así como de sus familias, o a su poca relevancia dada la falta de oportunidades al terminar la escuela. Esto ha motivado intensos debates que ameritan escenarios para su argumentación y análisis.

\section{Redefiniciones y fortalecimiento de la relación escuela-familia}

En este aspecto hubo un descubrimiento mutuo. Los maestros conocieron más de cerca cómo vivían sus estudiantes, su entorno familiar, su hábitat, sus problemas; establecieron unas relaciones más estrechas con los padres, madres y acudientes en general: 
Lo virtual les abre la casa a los profesores, abre a una realidad que no conocíamos antes [...] retomar esto para replantear la práctica en: vínculo con familia, reconocer las voces de los niños, inequidad social, pensar la vida en familia, la salud tiene que estar vinculada a la escuela, la siembra [...] que nos enternezcamos (Entrevista Albarracín, 2021).

Los padres valoraron los saberes y la experticia que se necesita para enseñar, estuvieron al tanto de los contenidos y las metodologías de los maestros, participaron más activamente de decisiones que antes parecía no interesarles. También se acercaron más a la manera como sus hijos aprenden, supieron mas de sus intereses, de sus angustias o sus preocupaciones. Para algunos maestros se abrió una amplia gama de posibilidades al no ver a los padres de familia simplemente como destinatarios de información sobre lo que sucede con sus hijos:

El diálogo con las familias ha permitido cambiar el imaginario sobre la educación de los más pequeños, mientras en la presencialidad no estaban tan dispuestos a escuchar y ven que lo que han logrado los niños con una experiencia literaria no es tan simple (Entrevista Niño, 2021).

La relación con las familias adqurió un lugar protagónico. Enfatizan aspectos como el vínculo humano y afectivo a través de la virtualidad y el reconocimiento de sus nuevas realidades, el trabajo colaborativo entre maestros y familias, la realización de foros interactivos y el trabajo en montajes colaborativos, el trabajo en equipo con las familias para apoyar el esfuerzo de los padres y estudiantes por seguir en el proceso académico.

A diferencia de los imaginarios que asocian escuela con falta de interés de madres y padres de familia, son numerosas las respuestas de éstos referidas a su deseo de conocer y relacionarse con las actividades escolares de sus hijos, así como su intención de perpetuar esta relación al volver a la presencialidad. Los padres propusieron que se continúe en la escuela postpandemia con muchas de las prácticas que se dieron en modo virtual, con el propósito continuar con su vinculación al proceso educativo de los niños y jóvenes, también para informarse sobre los temas tratados en clase para poderles ayudar de una manera más eficiente a sus niños. Al respecto, dice un maestro:

Los otro es que definitivamente hay que buscar la manera efectiva de hacer alianzas con las familias, porque cuando estamos en la presencialidad nosotros mismos nos quejamos de que no están presentes, pero (...) nos encargamos de aislar a las familias. (...) Reflexionar sobre los modos de enseñar y hacer parte a las familias, permitir que se involucren en los procesos, dejar de verlos como los que sólo van a dar quejas y permitir que entren al aula de otras maneras (Sandra Milene Niño, citada por Álvarez, Unda, García y Orozco, 2021, p. 116).

\section{Volver a la escuela}

El cierre de las escuelas debido a la pandemia quebró de manera intempestiva y radical la gramática (Álvarez, 2020) que había identificado a la escuela moderna desde su invención hace más de cuatrocientos años. Dos aspectos centrales a esta gramática se vieron radicalmente alterados en casi todas las escuelas oficiales de Bogotá: (i) la presencia de los cuerpos desapareció y se vio sustituida por la introducción o intensificación del uso de ciertos materiales y tecnologías (con excepción de algunas zonas rurales, donde no fue tan marcado); (ii) se desdibujaron los tiempos y espacios escolares y éstos se 
superpusieron con las actividades domésticas y laborales en los hogares de estudiantes y maestros.

Son numerosas y muy variadas las lecciones que han dejado las experiencias de educación en tiempos de confinamiento (Meirieu, 2020; Butler, 2020; Díaz-Barriga, 2020), las que aquí registramos provienen del interior de la escuela, de sus mismos actores y se convierten en experiencias clave en relación con una pregunta de especial interés para la administración pública, para la comunidad educativa y para la sociedad en su conjunto ¿Cómo volver a la escuela?

Indagar en las lecciones aprendidas por los distintos actores de la comunidad educativa a partir de lo vivido durante el período de confinamiento se convirtió, desde el inicio, en un propósito central de este estudio: ¿qué nos dice este tiempo sobre la escuela?, ¿qué valoraciones hacen estudiantes, maestros, padres de familia, orientadores y directivos, sobre las prácticas realizadas?, ¿̇a cuáles emergencias le darían continuidad y qué cambiarian de la escuela presencial anterior a la pandemia, después de pasar por esta experiencia?. Nos referimos, como dice Tenti en sus Notas sociológicas para la educación escolar postpandémica (2020, p. 77), a esos cambios más o menos profundos que pueden haber ocurrido en los modos de ver "las cosas de la escuela".

Esta aproximación se acerca más a lo que "esta época nos dice sobre la escuela que tenemos”, y no pretende resolver metodológicamente la cuestión práctica de cómo regresar. Nos referimos a la variedad de aprendizajes, quiebres y experimentaciones, que al haberse producido durante la pandemia y el aislamiento, ya hacen parte del acumulado de saberes y experiencias de las escuelas públicas que bien vale la pena reconocer, fortalecer y ampliar.

Todos los temas tratados en el informe de esta investigación dan cuenta de las lecciones aprendidas, de los debates abiertos, de las propuestas para volver a la escuela. En este cierre nos referimos a algunos aspectos centrales.

\section{La escuela presencial es imprescindible}

Lo vivido durante este período ha conducido a un redimensionamiento del valor de la escuela y del maestro. Los padres se dieron cuenta de todo el trabajo que implica la enseñanza, se sorprendieron con lo que hacen los maestros para conseguir la atención de los niños. Se vio que la escuela presencial cumple un papel fundamental:

No se era tan consciente de la importancia de verse y encontrarse. Lo que más falta es el contacto. Conceptualmente yo ya tenía muy enraizado que el centro del trabajo pedagógico es la relación pedagógica, ese vínculo, esa relación, pero ahora lo siento [...] Entonces lo que más me ha costado es esa falta de contacto, de vernos, de escucharnos. Uno no era consciente que eso fuera tan importante en el trabajo como profe (Entrevista Cárdenas, 2021)

Lo que más extrañó al $68 \%$ de los estudiantes que respondió a la encuesta fue no poder encontrarse físicamente con sus compañeros y maestros. Con pocas pero interesantes excepciones que ameritan juiciosas aproximaciones pues nos hablan de niños que consideran que no les afectó dejar de asistir a la escuela, los estudiantes narraron cómo empezaron 
a añorarla y redimensionaron cuanto vale el trabajo del maestro; narraron sus experiencias de no estar físicamente allí, de no encontrarse con compañeros y maestros, a veces en circunstancias difíciles por temas como la pobreza o la falta de trabajo que se vive con tanto dramatismo:

Se necesita la escuela presencial como escenario de socialización; además de ser un escape a las realidades que viven los jóvenes en sus casas, mejor dicho, en la casa se viven infiernitos (Entrevista Romero-Contreras, 2021).

La escuela en la práctica quedó dependiendo del maestro. A veces se sintió cansado, muchas veces desconcertado, pero fueron inmensos los esfuezos para ensayar e inventar, nadie sabía cómo enfrentar esta nueva realidad. Por supuesto que hubo dificultades pues esta educación mediatizada no reemplaza la escuela. Este planteamiento lo encontramos en muchas entrevistas, fueron contundentes, casi como un grito ¡Hay que garantizar el retorno a la presencialidad!

La escuela tiene un papel social y el maestro juega un rol definitivo en la educación de niños, niñas y jóvenes. Ingresar al mundo más allá de los referentes familiares o locales, de la información que brindan los medios de comunicación y las tecnologías; acceder a otros códigos de la cultura, modos de entender y estar en el mundo. La escuela quedó en suspenso porque su temporalidad se mezcló con la vida cotidiana y con la vida laboral. Lo que aporta la escuela presencial a la vida de los niños es la estructuración de espacios que, además de los saberes escolares, les permiten habitar el mundo con otros.
Aun cuando las tecnologías han sido decisivas para sostener el vínculo, diversificar las prácticas y permitir ciertos aprendizajes, queda como inquietud lo que significa el tiempo-espacio escolar en relación con el tiempo-espacio mediado por las tecnologías. Y la pregunta por la importancia del diálogo genuino entre un maestro y un grupo de estudiantes como el que se da en la presencialidad, de la conversación sostenida, donde la réplica y la explicación son posibles de una manera fluida. En la escuela hay una relación vinculante entre el maestro y los estudiantes que no se reduce a lo académico y se da en la interacción inmediata en la práctica pedagógica: mirar a los ojos, sentir al otro, corregir inmediatamente el error y alegrarse con sus avances, escuchar las voces de los niños no mediadas por la familia:

El sólo hecho de no estar interactuando directamente con los niños, es una diferencia grande, no podía tener el conocimiento de lo que estaba pasando con el desarrollo de los niños y las niñas, de sus conquistas. No es como uno lo vive en lo presencial que uno va siguiendo día a día sus conquistas o cuando retroceden, acompañamos, sostenemos la vivencia, eso es lo que extrañé (Entrevista Niño, 2021).

\section{Podemos cambiar: giros en la cultura escolar y las prácticas pedagógicas de los maestros}

Como hemos señalado, durante la pandemia la escuela se movilizó y tuvo la capacidad de hacerse sentir de múltiples maneras. Aquí queremos destacar este tipo de movimiento a veces sutil y silencioso, 
otras más generalizado, a veces desde las márgenes. Nos referimos a la capacidad de interrogar y problematizar las propias prácticas, el impulso para experimentar nuevas rutas.

Resulta muy valioso descubrir tantas iniciativas interesantes, algunas ya venían en camino y tuvieron capacidad de reconfigurarse para atender la situación de no presencialidad, otras surgieron y tomaron impulso con la pandemia. Todas ellas han puesto en evidencia que sí es posible cambiar hábitos, tradiciones y costumbres. Y contribuyen a pensar que al regreso, en la presencialidad, esto puede acentuarse. Pero también puede diluirse y desaparecer si nos dejamos vencer por la inercia de las culturas institucionales o de ciertas lógicas de gestión y administración de las escuelas. Para que esto no suceda, para continuar animando todos esos impulsos, se requiere una decisión, una voluntad que incluye la definición de planes, políticas y sobre los temas planteados por los integrantes de las comunidades educativas.

Así lo ameritan cada una de las experiencias descritas en las entrevistas: el trabajo colectivo en la escuela; los interrogantes sobre los currículos, las mallas curriculares y sus relaciones con las prácticas, sobre la organización escolar por disciplinas o áreas; la definición conjunta de prioridades y objetivos; la flexibilización y redefinición de las prácticas pedagógicas, el uso de las tecnologías y los dilemas éticos que se presentan en relación con los estudiantes que no tienen acceso; los sistemas de evaluación, la redefinición de relaciones entre las escuelas y las madres y padres de familia.

Todos estos desplazamientos en las prácticas pedagógicas de los maestros, están mostrando que las for- mas de trabajo en la escuela y los contenidos tradicionales de los currículos sí se pueden replantear. Los procesos y valoraciones de esta experiencia parecen confirmarlo. En palabras de una directiva: "Esta situación nos sacó de la zona de confort e hizo que los maestros florecieran y sacaran su garra para innovar" (Entrevista L.Peñuela González, citada por Álvarez, Unda, García y Orozco, 2021, p. 51).

Los debates están abiertos y muestran una escuela viva y deliberante que se pregunta por las prácticas institucionales de discriminación o exclusión como manuales de convivencia y prácticas de disciplinamiento, gobierno escolar, manejo del lenguaje, formas de ejercer la autoridad. También se pregunta por el reconocimiento de las diferencias y la atención de las desigualdades, los criterios y modalidades de evaluación, los modos de ver y de vincular a los padres de familia, en fin, los modos de pensar y de hacer escuela.

Podemos cambiar, continuar el camino de la experimentación a través de formas novedosas de enseñanza. Los maestros se refirieron a la posibilidad de continuar trabajando en equipos interdisciplinarios, los padres de familia propusieron continuar trabajando por proyectos, los estudiantes valoraron fortalecer el arte, el trabajo con el cuerpo y la enseñanza práctica. Se planteó la importancia de fortalecer la lectura, la literatura, la escritura creativa. Se recomendó implementar salidas pedagógicas y trabajar vinculados con el territorio.

El reconocimiento, la visibilización y los intercambios de experiencias pedagógicas son un deseo que expresan con insistencia los maestros después de haber experimentado todo lo que han podido crear. En el informe hay un listado de experiencias que compartieron con generosidad durante este estudio. 
Esa riqueza de trabajos que hay que recoger en algún momento, es la contracara de algo que se dice con frecuencia sobre las precariedades y no sobre la potencia del maestro y de la escuela.

Queda mucho por investigar. Esta pandemia ha trastocado, entre muchas otras estructuras, la gramática profunda de la escuela. Debemos acercarnos con audacia a esta realidad para valorar lo que ha sucedido durante la ausencia de las aulas y aprender de la experiencia de manera que se potencie la capacidad que han tenido las comunidades educativas, y en particular los maestros, para responder creativamente a esta insólita y desafiante situación ¡Una nueva escuela está por venir! 


\section{Referencias}

Albarracín, R. (2021). Entrevista a maestra. Estudio exploratorio: Valorar la experiencia, volver a la escuela. UPN, SED.

Entrevista maestra Nathalie Perez; citada por Álvarez, A., Unda, M. P., García, N. O., Orozco, J. H. (2021). Informe cualitativo de las prácticas pedagógicas realizadas por la comunidad educativa de los colegios oficiales de Bogotá, durante el confinamiento preventivo por la Covid-19. UPN, SED. https:// www.valorarlaexperiencia.com/resultados

Álvarez, A. (2020). La gramática del saber escolar. TD- Educação Temática Digital, v22, n4, 820-836. https://doi.org/10.20396/etd.v22i4.8660138

Braílovsky, D. (2020). Ecos del tiempo escolar. En: VV.AA. Pensar la educación en tiempos de pandemia. Entre la emergencia, el compromiso y la espera (pp. 149-161). Unipe.

Butler, J. (2020). El capitalismo tiene sus límites. En: VV.AA. Sopa de Wuhan. Pensamiento contemporáneo en tiempos de pandemias (pp. 59-65). ASPO.

Cárdenas, A. A. (2021). Entrevista a maestro. Estudio exploratorio: Valorar la experiencia, volver a la escuela. UPN, SED.

Cardini, A., Bergamaschi, A., D’Alessandre, V., Torre, E., Ollivier, A. (2020). Educar en pandemia: entre el aislamiento y la distancia social. Banco Interamericano de Desarrollo. https://doi. org/10.18235/0002494
Díaz-Barriga, A. (2020). La escuela ausente, la necesidad de replantear su significado. En: VV.AA. Educación y pandemia. Una visión académica (pp. 19-29). UNAM.

Diker, G. (2020). III Jornadas Nacionales de Formación Docente. Encuentro virtual "Narrar, sistematizar, investigar las prácticas. Las prácticas docentes en la continuidad pedagógica". Argentina, INFoD, https://www.youtube.com/watch?v=6O5-GQ38eJk

Fundación Chile. (2020). Informe de resultados. Encuesta \# Vinculando aprendizajes. Indagación sobre estrategias de los docentes y apoyos requeridos para la educación a distancia en contexto de crisis sanitaria.

Guzmán, M. F. (2021). Entrevista a maestra. Estudio exploratorio: Valorar la experiencia, volver a la escuela. UPN, SED.

López, J. P. (2021). Entrevista a maestra. Estudio exploratorio: Valorar la experiencia, volver a la escuela. UPN, SED.

Meirieu, P. (2020). La escuela es un lugar para construir en común. [Entrevista de X. Molénat y N. Nahapétian] http://www.mcep.es/2020/06/10/ la-escuela-es-un-lugar-para-construir-en-comun-philippe-meirieu/

Narodowski, M., y Campetella, D. (2020). Educación y destrucción creativa en el capitalismo de pospandemia. En: VV.AA. Pensar la educación en tiempos de pandemia. Entre la emergencia, el compromiso y la espera (pp. 43-52). Unipe. 
Niño, S. M. (2021). Entrevista a maestra. Estudio exploratorio: Valorar la experiencia, volver a la escuela. UPN, SED.

OISTE - Observatorio Interuniversitario de Sociedad, Tecnología y Educación. (2020). Resultados preliminares de la encuesta sobre enseñanza y aprendizaje en tiempos de cuarentena. http://noticias. unsam.edu.ar/2020/6/10/resultados-preliminares-de-la-encuesta-sobre-ensenanza-y-aprendizaje-en-tiempos-de-cuarentena/

Pinau, P. y Ayuso, M. L. (2020). De saneamientos, trancazos, bolsitas de alcanfor y continuidades educativas: brotes, pestes, epidemias y pandemias en la historia de la escuela argentina, En: VV.AA. Pensar la educación en tiempos de pandemia. Entre la emergencia, el compromiso y la espera (pp. 1932). Unipe.

ProBogotá, Centro Nacional de Consultoría, Universidad de los Andes. (2021). Covid-19 y educación en Bogotá: implicaciones del cierre de colegios y perspectivas para el 2021. https://egob.uniandes. edu.co/images/noticias/20210128-Primeros-Resultados.pdf

Pueyo, J. (2018). ¿Sustituir al profesor por tecnología? Asociación Educación Abierta. Disponible en: https://educacionabierta.org/sustituir-al-profesor-por-tecnologia/
Rodríguez, H. M., Estrada, J. C., Naranjo, L. C. (2020). Retos de la escuela en medio de la crisis pandémica. Revista Educación y Cultura, v.138, pp. 44-49.

Romero-Contreras, D. M. (2021). Entrevista a maestro. Estudio exploratorio: Valorar la experiencia, volver a la escuela. UPN, SED.

Rosero, J. (2021). Entrevista a maestro. Estudio exploratorio: Valorar la experiencia, volver a la escuela. Bogotá: UPN, SED.

SED. (2020). Primer informe de seguimiento y evaluación de la Estrategia Aprende en casa. Período comprendido entre 26 de marzo y 15 de abril de 2020. Bogotá: SED - Dirección de Evaluación de la Educación.

Tenti-Fanfani, E. (2020). Educación escolar post pandemia. Notas sociológicas. En: VV.AA. Pensar la educación en tiempos de pandemia. Entre la emergencia, el compromiso y la espera (pp. 71-83). Buenos Aires: Unipe.

VV.AA. (2020). Confinados en las pantallas. La escuela vira(l).Aula de Humanidades, Grupo Historia de la Práctica Pedagógica, GHPP. 